\title{
Evolution and Identification of Aquatic Macroinvertebrates Like Bioindicators of Water Quality
}

\section{Evolución e Identificación de los Macroinver- tebrados Acuáticos Como Bioindicadores de la Calidad de Agua}

\author{
Liz Ortiz Contreras, Bori Ortega, Patricio Méndez, and Patricio Tierra
}

I International Congress of Science and Technology Morona Santiago-CICTMS 2020

Corresponding Author:

Liz Ortiz Contreras

liz.ortiz@espoch.edu.ec

Published: 29 August 2021

Production and Hosting by

Knowledge E

(c) Liz Ortiz Contreras et al. This article is distributed under the terms of the Creative Commons Attribution License, which permits unrestricted use and redistribution provided that the original author and source are credited.
Escuela Superior Politécnica de Chimborazo (Espoch) Extensión Morona Santiago, Facultad de Ciencias, Macas, Ecuador

\section{Abstract}

This bibliographic review work presents a compilation of the bioindicators of water quality, its main advantages and disadvantages; the types of bioindicators are detailed, for example bacteria, protozoa, phytoplankton, macrophytes, fish and aquatic macroinvertebrates. It is important to know how the collection of macroinvertebrates is carried out, in the case of environments with running or sleeping waters, not very deep, a net is used, while for environments with currents or calm but deep waters, the use of dredgers is it is recommended that, after collection, it should be kept in appropriate containers until the types of bioindicators that were collected can be identified with the help of a microscope. It can be said that the use of aquatic macroinvertebrates as indicators of water quality is very widespread throughout the world, so their evolution has increased as they already have taxonomic species determined for different quality states. That is why the objective of this research is to know the evolutionary importance of aquatic macroinvertebrates as indicators of water quality, at the same time the advantages and disadvantages of the use of bioindicators, compared to physical-chemical methods.

Keywords: Bioindicator, macroinvertebrates, evolution, water quality, monitoring

\section{Resumen}

El presente trabajo de revisión bibliográfica muestra una recopilación de los bioindicadores de la calidad del agua, sus principales ventajas y desventajas; se detalla los tipos de bioindicadores por ejemplo bacterias, protozoos, fitoplancton, macrófitas, peces y macroinvertebrados acuáticos.

La importancia de este artículo radica en la recolección de macroinvertebrados, en el caso de ambientes con aguas corrientes o dormidas no muy profundas, se utiliza una red, mientras que en ambientes de aguas corrientes o tranquilas pero profundas, se recomienda el uso de dragas.

Luego de la recolección se debe mantener en envases apropiados, hasta que con la ayuda de un microscopio se pueda identificar los tipos de Bioindicadores que se colectó.

Se puede decir que el uso de los macroinvertebrados acuáticos como indicadores de la calidad del agua está generalizándose en todo el mundo, por lo que su evolución ha incrementado al tener especies taxonómicas determinadas para diferentes estados de calidad.

Es por ello que el objetivo de esta investigación es dar a conocer la importancia evolutiva que tienen los macroinvertebrados acuáticos como indicadores de la calidad del agua, así como las ventajas y desventajas que presenta la utilización de bioindicadores, en comparación con métodos físico-químicos. 
Palabras Clave: Bioindicador, macroinvertebrados, evolución, calidad del agua, monitoreo.

\section{Introducción}

Los sistemas hídricos están sometidos a constante movimiento de agua en donde se lleva consigo material particulado, sustancias provenientes de varias industrias o actividades antropogénicas [1], incluso sustancias de origen natural, las cuales consiguen ocasionar una sustancial alteración dentro de la biota que existe en los diferentes efluentes hídricos [2].

Para mitigar estas alteraciones existen estudios que confirman que la presencia de bosques en zonas de ribera ayuda a amortiguar el impacto negativo que generan las prácticas ganaderas sobre las microcuencas y mejoran los servicios ambientales que estas prestan [3].

Para determinar la calidad del agua, se debe realizar monitoreos físico-químicos o biológicos.

Las variables físico-químicas nos darán información de un lugar de referencia en un determinado tiempo [4], debido a que realiza una correlación entre los parámetros del agua y las actividades realizadas por el ser humano [5].

Otro factor que influencia en algunos parámetros físico-químicos, sobre todo en la temperatura, conductividad eléctrica y los sólidos totales disueltos, es el gradiente altitudinal [6].

Por otro lado, tenemos el análisis biológico del agua, el cual nos proporcionará un resultado más exacto de lo que sucede en un río, caudal, estuario, etc. [7].

En general los macroinvertebrados acuáticos constituyen un importante componente de la estructura en la comunidad biológica asociada a ambientes lóticos, pues desempeñan un rol crítico en el ecosistema, a través del mantenimiento de la estabilidad de la red trófica y balance de los ciclos biogeoquímicos [8, 9].

\section{Metodología}

Se evaluó la información de varios investigadores y de varias revistas como Scielo, Dialnet, Scopus y Redalyc.

Las principales palabras claves utilizadas para la búsqueda de la información fueron: calidad del agua, bioindicadores y macroinvertebrados, con un total de 80 artículos recopilados, publicados desde el año 2010 al presente, de los cuales 39 de estos artículos fueron los más relevantes.

En el texto se describen temas tales como los bioindicadores, sus ventajas y desventajas; organismos utilizados como bioindicadores entre ellos: bacterias, protozoos, fitoplancton, macrófitas, peces y macroinvertebrados acuáticos, profundizando en su recolección y evolución. 


\section{Desarrollo y Discusión}

\subsection{Biondicadores}

Se desarrollan de acuerdo al medio donde viven, pueden variar en relación con las alteraciones ambientales [10], es decir, las respuestas biológicas son observadas frente a una perturbación ecológica y están referidos como organismos o sistemas biológicos [11] que sirven para evaluar variaciones en la calidad ambiental [12]. La sensibilidad propia de cada uno de los organismos a la contaminación se utiliza para asignarle un valor de un índice biológico [13].

\subsubsection{Ventajas que presentan los bioindicadores}

Una de las principales ventajas que presentan los bioindicadores, es que, al poseer ciclos de vida largos, brindan un amplio conocimiento de los contaminantes a lo largo del tiempo, pues poseen diferentes tipos de respuestas a los gradientes ambientales. Mencionando que el muestreo se realiza de una forma sencilla y económica [14].

En conclusión, se puede decir que gracias a los procesos de acumulación de los organismos biológicos se pueden obtener panoramas completos de la interacción y efectos de los contaminantes en los ecosistemas y poblaciones acuáticas, características que no se pueden obtener con los análisis físico-químicos [15].

\subsubsection{Desventajas que presentan los bioindicadores}

Según (Springer, 2010), 'Una limitación que presenta este tipo de monitoreo es justamente al momento de determinar la calidad de agua para consumo humano, porque no necesariamente detecta la presencia de patógenos o condiciones químicas potencialmente peligrosas para la salud humana'. La evaluación de la calidad ambiental ya sea del agua, aire o suelo, con bioindicadores es un procedimiento que no nos permite cuantificar algunas variables, donde se hace necesarios estudios adicionales que rectifiquen la relación entre variables bióticas y abióticas; ya que el procedimiento indirecto requiere se demuestre la relación causa-efecto entre variables. Los bioindicadores no generan datos cuantitativos, por lo que no se compara con métodos físico químicos [16].

\subsection{Organismos utilizados como bioindicadores}

Los organismos que se utilizan como bioindicadores de la calidad del agua son las Bacterias, protozoos, Fitoplancton, macrófitas, peces y macroinvertebrados acuáticos.

\subsubsection{Bacterias}

Este tipo de indicadores se utiliza especialmente para determinar la calidad de los cuerpos hídricos receptores de descargas provenientes de aguas de uso doméstico 
y que pueden conllevar a la propagación de enfermedades [17], por lo cual es necesario establecer los índices de contaminación fecal mediante el conteo de coliformes, estreptococos, etc. [15].

\subsubsection{Protozoos}

Los protozoos son protistas microscópicos que generalmente son unicelulares, poseen un flagelo o cola [18], lo que les permite poseer movilidad, la mayoría de estos microrganismos son aeróbicos heterótrofos, sin embargo, existen algunos que son anaeróbi$\cos [19]$.

\subsubsection{Fitoplancton}

Al hablar de Fitoplancton nos referimos a la comunidad planctónica, con el predominio de: diatomeas y dinoflagelados, siendo estos representantes de la cadena alimenticia, pues se sabe que la productividad primaria no solo es importante para el equilibrio y regulación de los ecosistemas, sino también para un desarrollo sustentable [20, 21].

Este grupo de bioindicadores es importante pues responde rápidamente a los cambios que pueden ocurrir en las masas de agua por procesos naturales o antrópicos.

\subsubsection{Macrófitas}

Debido a la habilidad que tienen las macrófitas acuáticas para asimilar contaminantes (cloruros, sulfatos, nitratos, carbonatos, amoniaco), estas se han empleado en la detección y remoción de sustancias en efluentes de aguas residuales domesticas e industriales [22].

\subsubsection{PECES}

Son considerados un grupo de bioindicadores fáciles de muestrear por cualquier grupo interesado, al presentar una gran movilidad dentro del ecosistema acuático debido a que son capaces de huir de la contaminación y regresar cuando esta ha desaparecido, es decir, que cuando existe una gran densidad de especies de peces denota un ecosistema poco alterado [15]

\subsubsection{Macroinvertebrados acuáticos}

Se denominan macroinvertebrados acuáticos aquellos invertebrados con un tamaño superior a $500 \mu \mathrm{m}$, entre los que se incluyen animales como esponjas, planarias, sanguijuelas, oligoquetos, moluscos o crustáceos, entre los que se encuentran los cangrejos [23]. 
Los géneros de insectos acuáticos que se destacan, por su abundancia y distribución, son los siguientes: efemerópteros, plecópteros, odonatos, hemípteros, coleópteros, Tricópteros y dípteros [7]

\subsection{Recolección de macroinvertebrados}

Para obtener el conteo de macroinvertebrados por unidad de área se utiliza la red 'Surber', la misma que esta provista de un marco metálico, el cual sujeta una red de nylon muy fina donde se quedan atrapados los bichos luego de remover manualmente y contra corriente el sustrato que se encuentra dentro del marco metálico [24].

Una vez realizado la identificación con ayuda de las clases taxonómicas y la experiencia del investigador, se procede a guardar definitivamente los macroinvertebrados identificados en frascos de vidrio muy pequeños con alcohol al $90 \%$ para su preservación definitiva [24].

Estos animales deben estar correctamente identificados con una etiqueta en el interior del frasco, la misma que deberá contener la información correspondiente al sitio de colección, la fecha, condiciones del lugar donde fue colectado y su respectiva clasificación taxonómica [25].

\subsection{Evolución de los macroinvertebrados}

Se ha discutido sobre el nivel taxonómico más adecuado para estudios de bioindicadores, si bien es cierto que el nivel preferible sería el de especie, la taxonomía de ciertos grupos hace el trabajo prácticamente inviable en muchos países, en gran parte por el coste económico que ello comporta [26]. Especialmente en los dípteros, el trabajo de preparación e identificación, incluso a nivel de género, permite que su estudio sea económicamente muy costoso. Por ello un buen equilibrio entre calidad de los resultados y tiempo requerido para obtenerlos se da utilizando como nivel taxonómico la familia [27].

A lo largo del proceso de investigación se ha logrado identificar que las especies Atopophlebia, Hagenulopsis, Hydrosmilodon correspondientes al género Ephemerópteros [28] y las especies Plectrocnemia geniculata, Rhyacophila denticulata, Sericostoma selysii del género Tricópteros [29] son muy sensibles a la contaminación, en cambio que los Plecópteros son bioindicadores muy buenos para aguas sin contaminación [30].

Por otro lado, los Dípteros han sido identificados en su gran mayoría en aguas totalmente contaminadas, en aguas que tienen descargas domésticas y comerciales totalmente directas.

La familia Chironomidae ha sido caracterizada como tolerante a la contaminación debido a sus hábitos alimentarios saprófagos y en consecuencia su presencia está asociada a altas concentraciones de materia orgánica y bajos niveles de oxígeno. 
Las quebradas andinas albergan una significativa porción de la diversidad del orden Coleóptera en Sudamérica, de tal manera que pueden representar más del 50\% de la fauna conocida a nivel genérico en América del Sur [31].

Por su parte, los Tricópteros estuvieron bien representados por larvas y pupas, las cuales viven en diversos ambientes acuáticos y la mayoría construyen refugios fijados al sustrato o refugios portátiles de una variedad de formas y materiales [32].

Según (Springer, 2010), estos insectos acuáticos, además de ser diversos, son capaces de colonizar diferentes tipos de sustratos (roca, arena, grava, hojarasca), tanto en ríos de corrientes rápidas y turbulentas como en ríos de agua tranquila [33].

\section{Discusión}

El uso de los macroinvertebrados acuáticos como indicadores de la calidad de agua de los ecosistemas está generalizándose en todo el mundo.

Los beneficios del uso de herramientas biológicas y no solo las características fisicoquímicas del agua para la medida de su calidad han sido explicadas también en muchos libros, manuales, y forma parte de la legislación de muchos estados [34].

Particularmente interesante es el proceso abierto en la Unión Europea donde la indicación biológica es el núcleo de todo el sistema de monitoreo y evaluación de la calidad del agua de sus 27 países, dando incluso a luz a un nuevo concepto, el 'Estado Ecológico', y ello ha significado una revolución en la forma como los gobiernos europeos deben contemplar los indicadores biológicos de calidad del agua [35].

Sin embargo, en Latinoamérica se ha podido evidenciar la gran acogida a esta alternativa y eso se ha visto plasmado en 39 artículos científicos en los que hemos basado esta investigación, por lo tanto, su uso se recomienda en muchos de los protocolos de estudio de los países que los utilizan como indicadores de calidad biológica de forma reglamentada. En las circunstancias actuales, puede ofrecer más ventajas en América del Sur [36].

\section{Conclusiones}

La importancia evolutiva de utilizar macroinvertebrados, radica en que a lo largo del tiempo se ha podido identificar especies como bioindicadores de la calidad del agua, como por ejemplo las especies Atopophlebia, Hagenulopsis, Hydrosmilodon correspondientes al género Ephemerópteros y las especies Plectrocnemia geniculata, Rhyacophila denticulata, Sericostoma selysii correspondientes al género Tricópteros, son muy sensibles a la contaminación, en cambio que los Plecópteros son bioindicadores muy buenos para aguas sin contaminación.

La principal ventaja de trabajar con monitoreos biológicos es que, al tener ciclos de vida largos, los bioindicadores integran los efectos de la contaminación en el tiempo, lo que nos da una visión general de lo que está sucediendo en ese lugar, a diferencia de cuando realizamos monitoreos fisicoquímicos que nos indica los efectos de la contaminación que tiene el recurso hídrico únicamente en ese instante. 
Una desventaja de trabajar con bioindicadores es que no detecta presencia de patógenos o condiciones químicas peligrosas, es decir no es apto para determinar la calidad de agua para consumo Humano, mientras que por métodos físico químicos si se puede realizar dicha detección.

\section{References}

[1] Oscoz J, Campos F, Escala MC. Variación de la comunidad de macroinvertebrados bentónicos en relación con la calidad de las aguas. Limnetica. 2006;25(3):10.

[2] Gustavson S, Cosme L, Trama F. Macroinvertebrados bentónicos como indicadores de la calidad de agua en la microcuenca San Alberto, Oxapampa, Perú. 2013;03(02):16.

[3] Ramírez J, Giraldo L, Zuñiga M, Ramos B, Chrá J. Influencia de la ganadería en la comunidad de macroinvertebrados acuáticos en microcuencas de los Andes centrales de Colombia. Rev Biol Trop. 2018;66(3):1244.

[4] Vimos D. Influencia de las condiciones hidráulicas e hidrológicas en la variación espacial y temporal de las comunidades de macroinvertebrados acuáticos en los ríos altoandinos de cabecera al sur del Ecuador. Universidad Politécnica de Valencia; 2017.

[5] Aveiga A, Noles P, De la Cruz A, Peñarrieta F, Alcántara F. Variaciones físico-químicas de la calidad de agua del río Carrizal en Manabí. Enfoque UTE. 2019;10(3):30-41.

[6] Huanachín A, Huamantinco A. Composición y estructura de la comunidad de coleópteros acuaticos (Insecta: Coleoptera) a lo largo de un gradiente altitudinal, Cusco, Perú. Rev Peru Biol. 2018;25(2):131.

[7] Yépez Á, Yépez Á, Urdánigo J, Morales D, Guerrero N, TayHing C. Macroinvertebrados acuáticos como indicadores de calidad hídrica en áreas de descargas residuales al río Quevedo, Ecuador. Cienc y Tecnol. 2017;10(1):8.

[8] Peña S, Bohórquez H, Barrera A, Salamanca S, Jiménez D, Botello W. Macroinvertebrados como bioindicadores de la calidad del agua en la quebrada La Calaboza (Yopal, Casanare). Entre Cienc e Ing. 2019;13(25):14.

[9] Murillo S, Mendoza A, Restrepo E, Rodriguez M. Utilización de macroinvertebrados acuáticos como herramienta para determinar la calidad del agua en la quebrada Santo Tomás, municipio de Pensilvania, Colombia. Rev la Acad Colomb Ciencias Exactas, Físicas y Nat. 2018;42(164):212.

[10] Toledo M. Determinación de la calidad del agua acuáticos como bioindicadores en la microcuenca del río chimborazo. 2015;

[11] Herrera H. Camarones Penaeidos y carideos como bioindicadores de contaminación y su relación con variaciones en la Fisicoquímica de los sedimentos en la laguna Madre, Tamaulipas, México. Univ Autónoma nuevo León. 2019;52(81):88.

[12] Gamboa M, Reyes R, Arrivillaga J. Macroinvertebrados bentónicos como bioindicadores de salud ambiental. 2008.

[13] Castillo M. Determinación de la calidad de agua mediante indicadores físico, qupimicos y Biológicos en los bofedales de la reserva de producción de Fauna Chimborazo. 2018;

[14] Prat N, Ríos B, Acosta R, Rieradevall M. Los macroinvertebrados como indicadores de la calidad de agua. J Chem Inf Model. 2009;53(January):160.

[15] Padilla L. Caracterización de macroinvertebrados acuáticos como bioindicadores mediante índices para evaluar la calidad del agua en el rio Coca parroquia San José de Guayusa. 2017;

[16] Cutipa M. Evaluación de la calidad deaire en lazona de uso turpistico y recreativo- sector Huampal de Parque Nacional Yanachaga Chemilen Usando Xanthoria parietina, Como Bioindicadores. Univ Priv Tacna. 2019;

[17] Buñay M. Estudio de la calidad del agua de la micro cuenca del Río Tingo mediante la utilización de un índice de calidad del agua (WQI) y la identificación de macro invertebrados como bioindicadores, para el GAD Provincial de Chimborazo. Esc Super Politécnica Chimborazo. 2016;Bachelor:139.

[18] Ymas I, Revilla L, Prieto D. Evaluación de la contaminación de la Presa Ejército Rebelde, Ciudad de La Habana, Cuba, mediante el empleo de protozoos como bioindicadores. 2009;37-42.

[19] Beltrán T, Campos C. Influencia de microorganismos eficaces sobre la calidad de agua y lodo residual, planta de tratamiento de Jauja. Univ Nac del Cent del Perú. 2016;206.

[20] Saransig R. Estudio de la Calidad de Agua en los Afluentes de la Microcuenca alta del Río Guargualla para determinar las causas de la degradación y Alternativas de manejo. Esc Super Politécnica Chimborazo. 2009;45(1):1-19.

[21] Falero S. Variación espacio- temporal trimestral de fitoplancton marino en la plataforma albacora del lote Z1, Provincia de Tumbes-Tumbes,Perú. Univ Ricardo Palma. 2019; 
[22] Martelo J, Lara J. Macr'ofitas flotantes en el tratamiento de aguas residuales: una revisión del estado del arte. Ing y Cienc. 2012;8(15):221-43.

[23] Resh V. ¿Qué grupo es el mejor? Atributos de diferentes conjuntos biológicos utilizados en programas de biomonitoreo de agua dulce. Springer. 2008;131-8.

[24] Molano M, García D. Determinación del índice BMWP/Col, mediante la utilización de macroinvertebrados como bioindicadores de la calidad de agua, en el cauce del rio Guachicos, que surte el acueducto del municipio de Pitalito. J Chem Inf Model. 2019;53(9):1689-99.

[25] Yungán J. Estudio de la calidad de agua en los afluentes de la microcuenca del Río Blanco para determinar las causas de la degradación y alternativas de manejo. ESPOCH. Escuela Superior Politénica de Chimborazo; 2010.

[26] Yumbo K, lleer V, Espinoza W, Campos D, Castro R, Chirinos D. Determinación de la calidad de aguas mediante indicadores biológicos y físico-químicos en el Río Pajan, Manabí, Ecuador. Investigatio. 2018;(10):32-40.

[27] González S, Ramírez Y, Meza A, Dias L. Diversidad De macroinvertebrados acuáticos y calidad de agua de quebradas abastecedoras del municipio de Manizales. Boletín Científico Cent Museos Mus Hist Nat. 2012;16(2):135-48.

[28] Flowers R., De la Rosa C. Ephemeroptera. Greenl Entomofauna. 2015;83-4.

[29] Basaguren A, Orive E. Los insectos tricopteros como indicadores de la calidad del agua de los rios de Bizkaia, cuenca del Nerbion. Kobie Ciencias Nat. 1991;20:39-44.

[30] Ramírez A, Gutiérrez P. Estudios sobre macroinvertebrados acuáticos en América Latina: Avances recientes y direcciones futuras. Rev Biol Trop. 2014;62:9-20.

[31] Zúñiga M, Chará J, Giraldo L, Serna A, Pedraza X. Composición de la comunidad de macroinvertebrados acuáticos en pequeñas quebradas de la región andina colombiana, con énfasis en la entomofauna. Dugesiana. 2013;20(2):263-77.

[32] Valerio M, Tafur C. Calidad de agua según los macroinvertebrados bentónicos y parámetros físicoquímicos en la microcuenca del río Tablachaca (Ancash, Perú). Rebiol. 2015;35(2):75-89.

[33] Rodríguez J, Rojas J, Gómez J. Ensamble de macroinvertebrados acuáticos y estado ecológico de la microcuenca Dalí-Otún, Departamento de Risaralda, Colombia. Hidrobiologica. 2016;26(3):359-71.

[34] Cuarán D, Ruiz K. Evaluación del estado trófico en relación a los parámetros físico-químicos y macroinvertebrados bentónicos del lago San Pablo, cantón Otavalo. Universidad Técnica del Norte; 2019.

[35] Meza A, Rubio J, Dias L, Walteros J. Calidad de agua y composición de macroinvertebrados acuáticos en la subcuenca alta del río Chinchiná. Caldasia. 2012;34(2):443-56.

[36] Machado V, Granda R, Endara A. Análisis de macroinvertebrados bentónicos e índices biológicos para evaluar la calidad del agua del río Sardinas, Chocó Andino ecuatoriano. Enfoque UTE. 2018;9(4):154-67.

[37] Springer M. Biomonitoreo Acuático. Rebiol. 2010;58(2):75-89.

[38] Camargo JAA. Estado actual y perspectivas en el empleo de la comunidad de macroinvertebrados bentónicos como indicadora del estado ecológico de los ecosistemas fluviales españoles. Ecosistemas Rev científica y técnica Ecol y medio Ambient. 2005;14(3):10.

[39] Springer M. Trichoptera. Rev Biol Trop. 2010;58(4):51. 